\title{
Lactobacillus plantarum LP-Onlly alters the gut flora and attenuates colitis by inducing microbiome alteration in interleukin-10 knockout mice
}

\author{
HONGQI CHEN $^{1}$, YANG XIA ${ }^{1}$, SIBO ZHU $^{2}$, JUN YANG $^{1}$, JING YAO $^{1}$, \\ JIANZHONG DI ${ }^{1}$, YONG LIANG ${ }^{1}$, RENYUAN GAO ${ }^{1}$, WEN WU ${ }^{1}$, YONGZHI YANG ${ }^{1}$, \\ CHENZHANG SHI $^{1}$, DESHENG HU ${ }^{1}$, HUANLONG QIN ${ }^{1}$ and ZHIGANG WANG ${ }^{1}$ \\ ${ }^{1}$ Department of General Surgery, Shanghai Jiao Tong University Affiliated Sixth People's Hospital, Shanghai 200233; \\ ${ }^{2}$ Department of Molecular and Cellular Biology, Cinoasia Institute, Shanghai 200438, P.R. China
}

Received October 25, 2016; Accepted June 15, 2017

DOI: $10.3892 / \mathrm{mmr} .2017 .7351$

\begin{abstract}
The association between inflammatory bowel disease (IBD) and gut microbes has been widely investigated. Our previous study demonstrated that Lactobacillus plantarum LP-Onlly (LP) applied as a probiotic altered the gut flora and attenuated colitis in interleukin (IL)-10 knockout (IL-10 ${ }^{-/}$) mice. In the present study, metagenome sequencing was performed to investigate the gut microbiome in IL-10-- mice and the influence of oral administration of LP on microbial composition. Metagenomics sequencing was performed to investigate the influence of IBD on the gut microbiome with and without LP treatment. The alteration of the abundances of various taxonomic and functional groups were investigated across these gut microbiomes. The present study demonstrates that Akkermansia muciniphila was significantly enriched in IL-10 ${ }^{-/-}$mice, and bacteroides were significantly increased following LP administration. In addition, the phylum Bacteroidetes and Firmicutes were significantly influenced by LP administration. Further characterization of functional capacity revealed that in the gut metagenomes of $\mathrm{IL}-10^{-/}$mice, genes encoding cell cycle control, replication, recombination, repair and cell envelope biogenesis were decreased, but intracellular trafficking, secretion, and vesicular transport were increased. The present findings indicate that the gut metagenome is associated with IBD, and oral administration of LP contributes to prevention of gut inflammation, providing insight into the treatment of IBD.
\end{abstract}

Correspondence to: Dr Huanlong Qin or Dr Zhigang Wang, Department of General Surgery, Shanghai Jiao Tong University Affiliated Sixth People's Hospital, 600 Yishan Road, Shanghai 200233, P.R. China

E-mail: hlqin10@126.com

E-mail: surlab@hotmail.com

Key words: Lactobacillus plantarum, colitis, interleukin-10 knockout mice, metagenomics

\section{Introduction}

The human gut harbors a wide range of microorganisms that determine the hemostasis of the host and enable various metabolic functions, such as biosynthesis of vitamins that humans are not born with (1). The gut microbiota are vital in host health development by interacting with the host (2). A general dysbiosis in microbiota composition and abnormal interactions in gut microbiota may result in various types of disorder. Current gut microbiota studies contribute to the understanding of the complex interactions between biological processes of the microbiota and host. These interactions characterize the underlying mechanism of the association between human health and gut microbiota.

Inflammatory bowel diseases (IBD) is characterized as a group of chronic gastrointestinal inflammation disorders, including Crohn's disease and ulcerative colitis (3). A recent study revealed that IBD is caused by the alterations in gut microbial communities and abnormal interactions between the immune system and the gut microbiota (4). A broad range of microbiota reside in the gastrointestinal tract, including the phylum Firmicutes, Bacteroidetes, Proteobacteria and Actinobacteria, which are dominant in the gut microbial community (5). Normally in a healthy gut, Firmicutes occupy $\sim 60 \%$ of the microbiota, whereas Bacteroidetes constitute $\sim 20 \%$ of the normal human microbiota $(6,7)$. However, the abundance of microbiota is imbalanced in IBD and the diversity is also reduced $(8,9)$. Microbiota dysbiosis of IBD includes the increased abundance of the phylum Proteobacteria and Bacteroidetes, while the phyla Firmicutes is decreased $(10,11)$. The microbiota composition is associated with gastrointestinal inflammation, therefore the majority of therapeutic strategies for IBD are focused on reconstructing the normal microbiota community of the host gut.

Probiotics are reported to benefit the host, and are non-digestible and fermentable (12). Functional studies of probiotics have been performed in the treatment of a series of inflammatory conditions, including ulcerative colitis and Crohn's disease (13-15). Via stimulating the growth of commensal flora, probiotics alter the composition of the 
intestinal microbes and enhance resistance to detrimental bacteria localization, therefore contributing to colitis reduction $(16,17)$. Administration of various probiotic strains has been identified as an effective treatment method for IBD (18). Our previous work demonstrated the administration of Lactobacillus plantarum LP-Onlly (LP) may attenuate inflammation of colitis in knockout (IL-10 ${ }^{-/}$) mice (19), however, the underlying mechanism remains unknown.

The aim of the present study was to reveal the alteration of gut microbiota under the influence of LP administration in colitis and clarify the underlying mechanism of LP treatment in experimental colitis. Metagenomic sequencing was performed to investigate the diverse microbiota in IL-10 deficient (IL-10 ${ }^{-/}$) mice with and without LP administration. In addition, a group of wild type (WT) mice and another group of mice with LP treatment (WT + LP) were sequenced to serve as a control. The abundance of microbiota in the LP treated mice $\left(\mathrm{IL}-10^{-/-}+\mathrm{LP}\right)$ and mice without LP treatment $\left(\mathrm{IL}-10^{-/-}\right)$was compared. De novo assembly revealed the taxonomic classification, and further characterized the functional activities of colitis and LP treatment in the gut microbiota of mice.

\section{Materials and methods}

Animals. Homozygous IL-10 ${ }^{-/}$mice (weight, $220 \pm 12 \mathrm{~g}$; age, 8 weeks; sex, female) generated on a $129 \mathrm{~Sv} / \mathrm{Ev}$ background $(n=12)$, and normal $129 \mathrm{~Sv} / \mathrm{Ev}$ controls $(n=12)$ (The Jackson Laboratory, Bar Harbor, ME, USA) were housed under specific-pathogen-free conditions (temperature, $25^{\circ} \mathrm{C}$; humidity, 70\%) in Shanghai Jiao Tong University Medical School (Shanghai, China). Mice were fed a standard sterile diet and filtered water ad libitum under a 12-h light/dark cycle. The animal studies were approved by the Ethical Committee of the Affiliated Sixth People's Hospital of Shanghai Jiao Tong University. Scoring of the disease activity index was performed by an individual blinded to the treatment.

Microbiome genomic DNA extraction and sequencing. Microbiome genomic DNA from mouse stools was prepared using a QIAamp Fast DNA Stool Mini kit (Cat No. 51604, Qiagen $\mathrm{GmbH}$, Hilden, Germany). All samples were sequenced in the Illumina HiSeq2000 instrument at SciLifeLab (Stockholm, Sweden) with up to 10 samples pooled in one lane. Libraries were prepared with a fragment length of $300 \mathrm{bp}$. Paired-end reads were generated with $100 \mathrm{bp}$ in the forward and reverse direction. Sequencing adapter sequences were removed with cutadapt (http://code.google.com/p/cutadapt/). The length of each read was trimmed using SolexaQA (http://solexaqa. sourceforge.net/) with the options ' $-b-p$ 0.05'. Read pairs with either reads $<35$ bp were removed with a custom Python script. The high-quality reads were then aligned to the human genome (National Center for Biotechnology Information; NCBI version 37) with Bowtie using '-n 2-1 35-e 200-best-p 8-chunkmbs 1024-X 600-tryhard'. This set of high-quality reads was subsequently used for further analysis.

Alignment to reference genomes and taxonomical analysis. A set of 2,797 microbial reference genomes were obtained from the NCBI and Human Microbiome Project $(20,21)$ on 02 August 2011. The reference genomes were combined into two Bowtie indexes and the metagenomic sequence reads were aligned to the reference genomes using Bowtie with parameters '-n 2-1 35-e 200-best-p 8-chunkmbs 1024-X 600-tryhard'. Mapping results were merged by selecting the alignment with fewest mismatches; if a read was aligned to a reference genome with the same number of mismatches, each genome was assigned half to each genome. The relative abundance of each genome was calculated by summing the number of reads aligned to that genome divided by the genome size. In each subject, the relative abundance was scaled to sum to one. The taxonomic rank for every genome was downloaded from NCBI taxonomy to assign each genome to a species, genus and phyla. The relative abundance for each taxonomical rank was calculated by summing the relative abundance of all its members.

Statistical analysis. The high-quality reads were used for de novo assembly with Velvet (22) into contigs (length, $\geq 500 \mathrm{bp}$ ) using 3 as the coverage cutoff and a kmer length of 31. To obtain long contigs with high specificity, parameter values for the kmer length and coverage cutoff were iteratively investigated to balance the total assembly length and the N50 value to be used in the final de novo assembly. Reads from each subject were used in separate assemblies and unassembled reads were subsequently used in a global final assembly. Genes were predicted on the contigs using MetaGeneMark (23). All genes were then aligned on the contigs with Bowtie using the above-mentioned parameters. The abundance of a predicted gene was calculated by counting the number of reads that align to the gene, and then the read counts were normalized by the gene length and the total mapped reads. The genes were annotated to the eggCOG database (24) with hidden Markov models (HMMs). Protein sequences for microbial orthologs were downloaded and aligned with MUSCLE (25). HMMs were generated with HMMer3 (26) for each KO mouse.

To determine the differential abundance of metagenomic features, an unpaired t-test was applied. Strains and genera with a relative abundance in any subject $>10$ were included in the analysis. The R package ade4 (27) (https://www.r-project .org/) using instrumental principal component analysis was used to determine the global analysis of species abundance between each group.

The relative abundance of a given taxon in a community was calculated as: Relative abundance $=\mathrm{a} / \mathrm{b} \times 100 \%$.

Where ' $a$ ' is the number of sequences assigned to the taxon and ' $b$ ' is the total number of sequences assigned to all the taxa in the community). Similar calculations were performed for relative abundance of a given gene, Clusters of Orthologous Groups (COG), COG category, Kyoto Encyclopedia of Genes and Genomes (KEGG) pathway and KEGG subcategory (28). Statistical enrichment of a given gene or COG between two data sets was determined by pairwise comparisons using two-tailed Fisher's exact test, with confidence intervals at 99\% significance and Benjamini-Hochberg correction. In all analyses, $\mathrm{P}<0.05$ was considered to indicate a significant difference.

\section{Results}

$L P$ treatment reduces inflammation in the mouse gut. To investigate the important role of LP in gut microbiota homeostasis, 
A

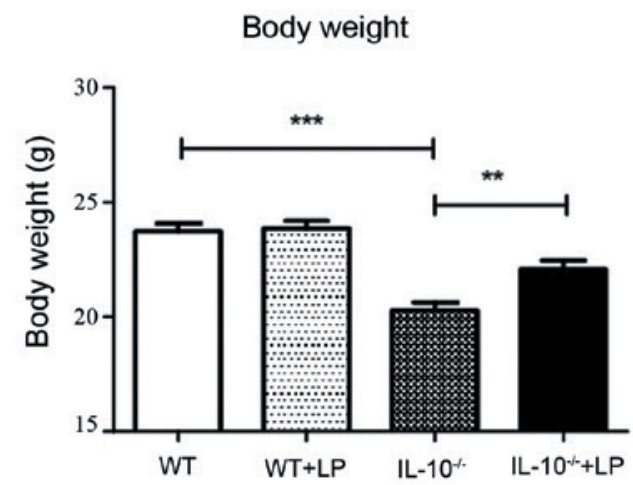

B

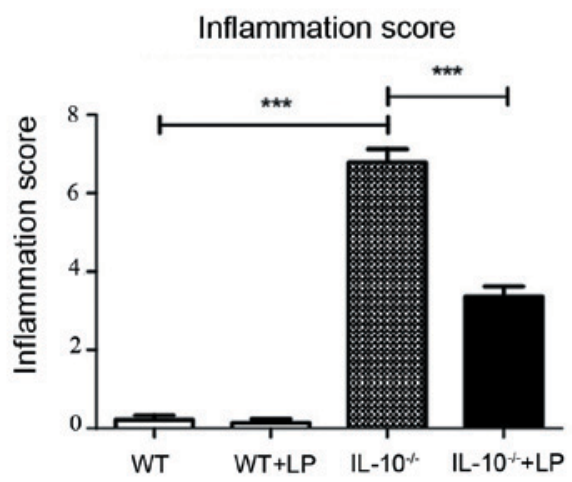

Figure 1. (A) Body weights and (B) inflammation score of IL-10 $10^{-/}$mice and IL-10 $10^{-/}$mice with LP treatment. ${ }^{* * *} \mathrm{P}<0.01$ and ${ }^{* * *} \mathrm{P}<0.001$. IL, interleukin; IL-10 ${ }^{-/}$, IL-10 knockout; WT, wild type; LP, Lactobacillus plantarum LP-Onlly.

four groups of mice models were established: WT (WT mice without LP treatment), WT + LP (WT mice with LP treatment), IL-10 ${ }^{-/}$(IL-10 ${ }^{-/-}$mice without LP treatment), and IL-10 $0^{-/}+$LP (IL-10 ${ }^{-/}$mice with LP treatment) $(n=6 /$ group). Consistent with a previous study (19), we observed that LP administration attenuated the inflammation syndrome of gut colitis. The body weight of IL-10 $10^{-/-}$mice decreased markedly after 4 weeks; however, the body weight of the IL- $10^{-/-}$mice with LP treatment was significantly greater than that of mice without LP treatment (Fig. 1A). Consistently, the inflammation score of IL-10 $10^{-/}$mice was significantly greater than that of IL-10 ${ }^{-1-}$ mice with LP feeding, and no inflammation syndrome was observed in the WT groups (Fig. 1B).

LP administration profoundly affected the gut microbiome. To clarify the influence of LP administration on the gut microbial environment in the gut of colitis mouse models, the gut metagenome of the four groups of mice was sequenced. A total of 411 million 101-bp paired-end clean reads were generated, the sequencing adapter and low quality reads were removed. The reads from mice genomes were identified and filtered. To reveal the composition of the gut microbiota, all the remaining reads were aligned to a catalog of 2,797 non-redundant NCBI microbial reference genomes (29). On average, $23 \%$ of the reads in a sample could be aligned to the reference genome, this ratio was close to the previous metagenome studies (29).

The principal component analysis confirms that the WT samples and IL-10 ${ }^{-/}$samples were differentiated by the abundance of microbial species. As presented in Fig. 2, samples of control group with LP administration were clustered together and the IL-10 $10^{--}+$LP samples were clustered in another group. However, the IL-10 ${ }^{-/}$samples without LP administration were dispersed, this may indicate that variation existed among the microbes in the most seriously inflamed gut samples.

To investigate the influence of inflammation on gut microbial communities and regulation of LP administration, the change of identified microbial abundance was compared between the different experiment groups. The species A. muciniphila and Parabacteroides distasonis were enriched in IL-10--mice, whereas Helicobacter hepaticus was enriched in the control groups (Fig. 3). Previous studies have confirmed the association between these three types of species and IBD.

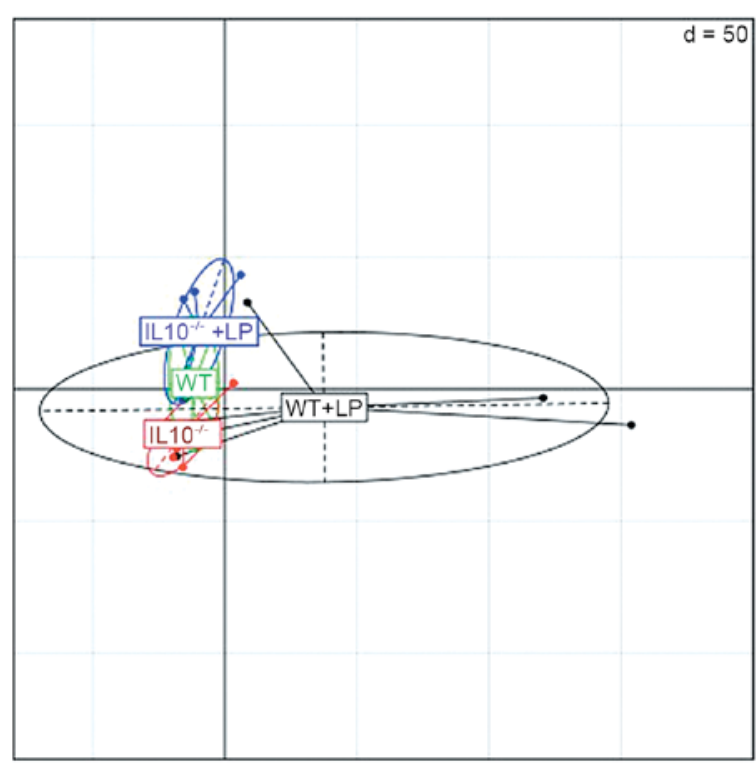

Figure 2. Principal component analysis of microbial species abundance among the four groups of samples. Green is WT mice $(n=6)$, black is WT mice with LP treatment, red is IL-10/- mice $(n=6)$, and blue is $\mathrm{IL}-10^{-/}$mice treated with LP ( $\mathrm{n}=6)$. WT, wild type; LP, Lactobacillus plantarum LP-Onlly; IL, interleukin; IL-10 ${ }^{-/}$, IL-10 knock out.

A. muciniphila exacerbates gut inflammation by disturbing host mucus homeostasis (30). However, the oral administration of $P$. distasonis has been reported as a novel therapeutic strategy for IBD (31). In addition, the H. hepaticus was associated with IBD.

L. plantarum is frequently used as a probiotic, and has been associated with the maintenance of intestinal homeostasis and modulation of the immune system. It regulates the quantity of pathogenic bacteria. Following L. plantarum administration, the abundance of three types of microbes, namely, Bacteroides uniformis, $P$. distasonis and Bacteroides salanitronis, were downregulated compared with the IL-10 $10^{--}$control groups. Bacteroides uniformis have been identified as essential members of gut microbiota, and are enriched in the gut of healthy individuals without IBD $(32,33)$. Furthermore, P. distasonis exerts beneficial 

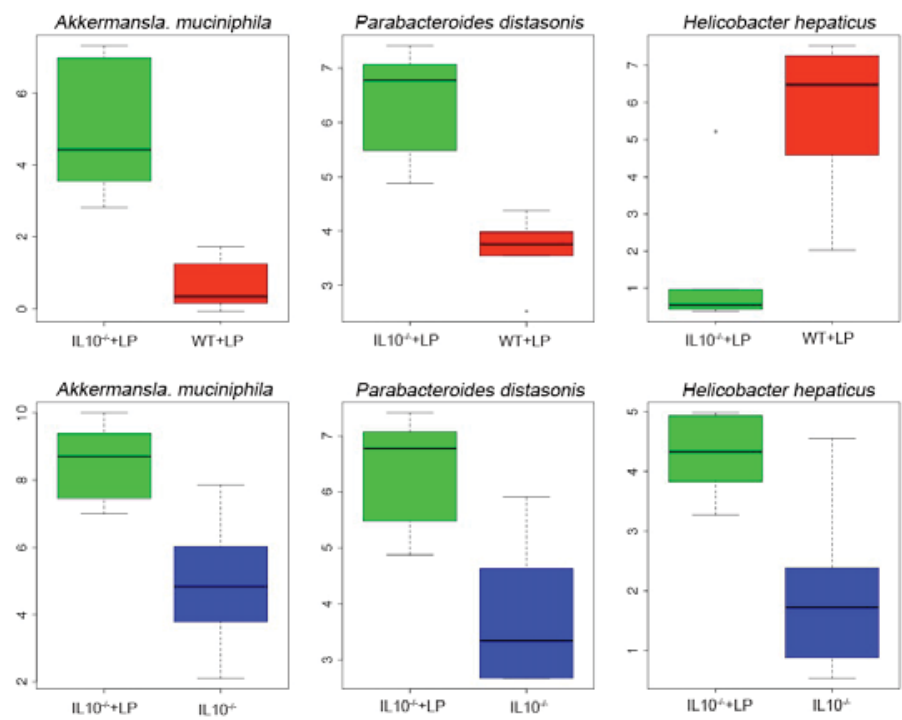

Figure 3. Abundance of genera and species that differ between IL-10/- and WT and the difference between IL-10/- mice with and without LP treatment. WT, wild type; LP, Lactobacillus plantarum LP-Onlly; IL, interleukin; IL-10 ${ }^{-/}$, IL-10 knock out.

A

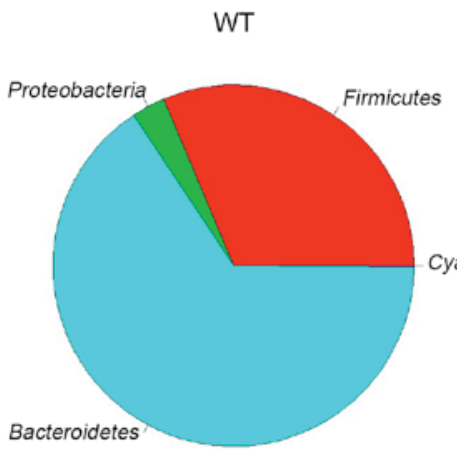

C

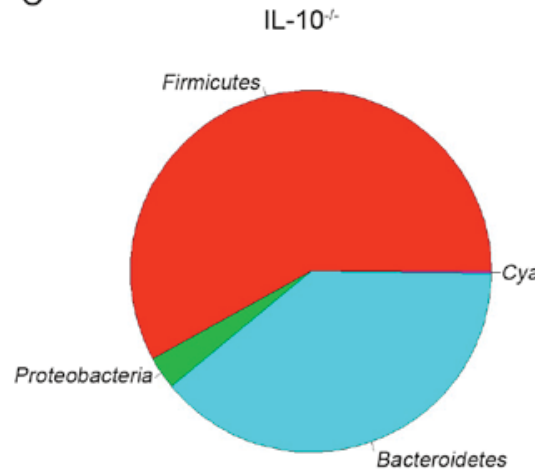

B

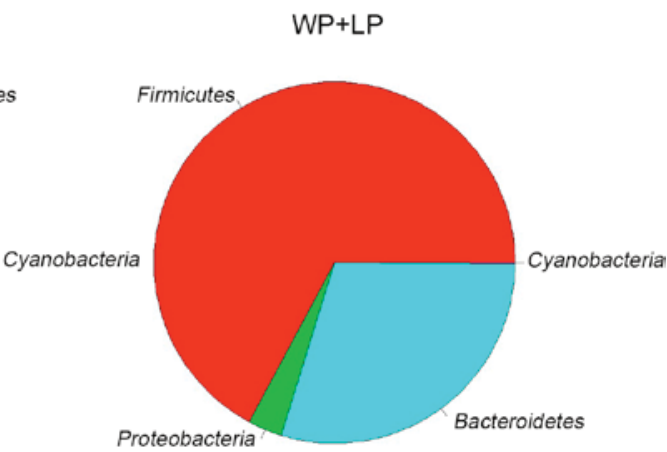

$\mathrm{D}$

$$
\mathrm{IL}-10^{-1+\mathrm{LP}}
$$

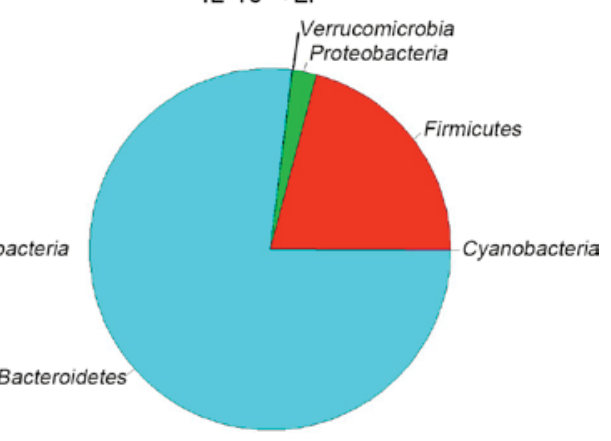

Figure 4. IL-10- 1 mice and LP treatment affect the proportions of different phyla. The composition of abundant bacterial phyla identified in the gut microbiota of (A) WT mice, (B) WT mice treated with LP, (C) IL-10-/mice (n=7) and (D) IL-10/- mice treated with LP. The undetected phyla are not presented in this figure. WT, wild type; LP, Lactobacillus plantarum LP-Onlly; IL, interleukin; IL-10 ${ }^{-/}$, IL-10 knock out.

effects on gut health; Kverka et al identified that the oral administration of $P$. distasonis attenuated the inflammation of IBD by modulation of immunity (31). To the best of our knowledge, our study is the first to report the association between Bacteroides salanitronis and IBD. These findings may indicate that the loss of probiotic in the IL-10 $10^{-/}$mice gut may aggravate the inflammation and that $L$. plantarum may increase the abundance of probiotic.
Taxonomic characterization in the mice gut microbiomes. To further identify novel genes in the mice gut metagenome and investigate the variation in IL-10 $0^{-/}$mice gut microbiota communities with and without LP administration, the de novo assembly was performed for the sequence data. In total 0.32 Gbp of contigs $>500$ bp were assembled with an N50 value of $0.8 \mathrm{kbp}$ and 5 as the coverage cutoff. Genes were predicted using the assembled contigs and 0.2 million non-redundant 
genes were identified. For the phylum level, as presented in Fig. 4, the gut inflammation and LP administration greatly affected the phyla proportion of Firmicutes and Bacteroidetes, as well as the abundance of various other phyla. This was consistent with previous studies, that the mice gut microbiome was greatly dominated by Firmicutes, Bacteroidetes and Proteobacteria. The ratio of Bacteroidetes/Firmicutes was markedly decreased in IL-10 $1 /$ mice, which may be associated with the inflammation of mice gut; however, following LP administration, the Bacteroidetes/Firmicutes ratio in IL- $10^{-/}+$LP group was increased compared with the IL-10 ${ }^{-1-}$ group, and was comparable with the control group. In addition, a small influence on Cyanobacteria and Proteobacteria was also observed in IBD in the current study. Furthermore, a total of 36 genera/phylum were affected by the inflammation and LP administration. The variation of the abundance of microbiomes in the four groups is presented in Fig. 5.

Functional activities of the mice gut microbiota. Gut microbial activities are associated with host physiological function, may influence metabolism and are a key factor in the inflammation state of IBD. Therefore, to characterize the functional activities of the mice gut microbiota, the predicted genes were functionally annotated to the eggCOG database and the relative abundance of COGs was assessed. The marked variation of COG abundance was observed between the three groups of mice gut microbiotas (Fig. 6). Twenty COG classes demonstrated significant differences in at least two groups of microbiomes. Numerous categories of COG were significantly decreased in the IL-10 $0^{-/-}$mice compared with the WT + LP groups, which included 'Cell cycle control, cell division', 'Amino acid transport and metabolism', 'Carbohydrate transport and metabolism', 'Transcription', 'Replication, recombination and repair', 'Cell wall/membrane/envelope biogenesis' and 'General function prediction only', while the abundance of these classes was increased in the LP administration groups of IL-10 $10^{-/}$mice. Furthermore, the abundance of categories 'Intracellular trafficking, secretion and vesicular transport' was significantly enriched in the IL $10^{-/-}$group.

\section{Discussion}

Probiotics have an important role in maintaining gut microbiota homeostasis, the imbalances in microbial communities may contribute to the gut disease of the host (14). Therefore, probiotics have been utilized as an effective treatment for gut disease. The onset and progression of IBD have been attributed to alteration of microbiota composition and the interaction between immune system and microbiota. Our previous work and other studies have demonstrated that probiotic treatment attenuates inflammation of colitis (19). In the current study, a colitis model was constructed using IL-10 $10^{-/}$mice. A metagenome sequence approach was conducted to investigate the effect of LP in colitis in IL-10 ${ }^{-/-}$mice. Weight loss was observed in the IL-10 $10^{-/}$mice compared with the control group and the inflammation score was particularly high. However, the inflammation score and weight loss were improved following LP treatment in IL-10 ${ }^{-/}$-deficient mice. This indicated that the colitis mice model had successfully been constructed, and LP administration is effective in the treatment of colitis.

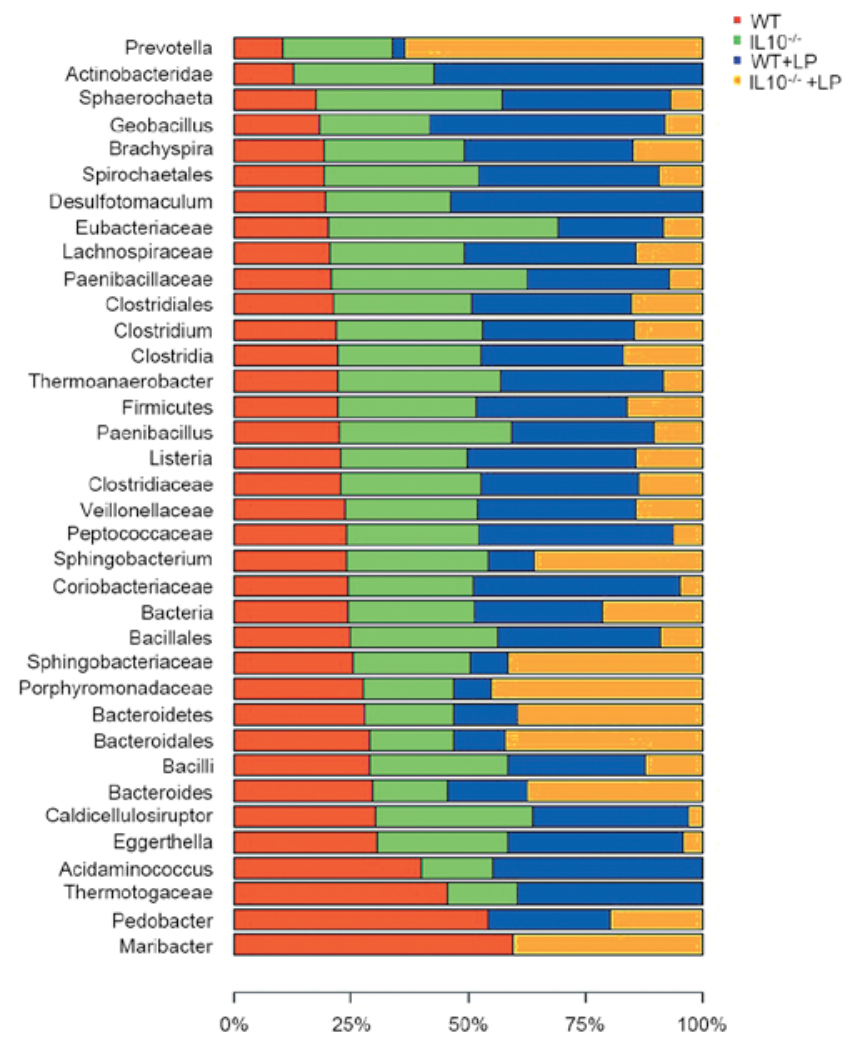

Figure 5. IL-10 $10^{-/}$mice and LP treatment affect the proportions of different genera. The composition of these genera significantly affected gut inflammation and were identified in the gut microbiota of IL-10 ${ }^{-/}$mice. Each column is set at $100 \%$ to illustrate the proportion of each genus among the different groups; the absence of any color indicates that the genus was not detected in this group of mice. WT, wild type; LP, Lactobacillus plantarum LP-Onlly; IL, interleukin; IL-10

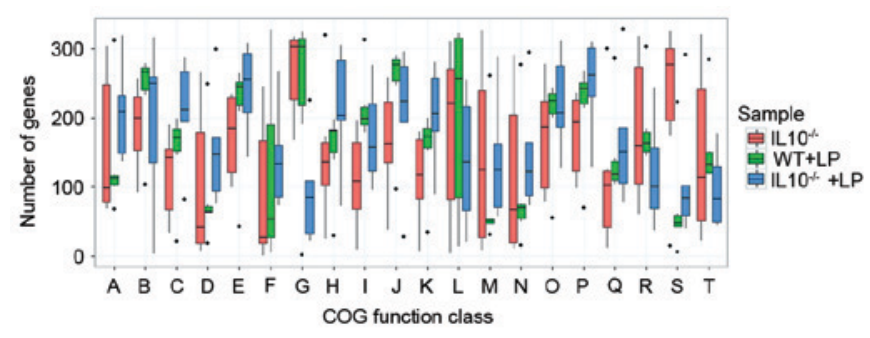

Figure 6. COGs of proteins affected by the IL-10 $1 /$ and LP treatments Occurrence of COGs of proteins according to the following COG functional categories: (A) Energy production and conversion; (B) Cell cycle control cell division; (C) Amino acid transport and metabolism; (D) Nucleotide transport and metabolism; (E) Carbohydrate transport and metabolism; (F) Coenzyme transport and metabolism; (G) Lipid transport and metabolism; (H) Translation, ribosomal structure and biogenesis; (I) Transcription; (J) Replication, recombination and repair; (K) Cell wall/membrane/envelope biogenesis; (L) Cell motility; (M) Posttranslational modification, protein turnover, chaperones; (N) Inorganic ion transport and metabolism; (O) Secondary metabolites biosynthesis, transport and catabolism; (P) General function prediction only; (Q) Function unknown; (R) Signal transduction mechanisms; (S) Intracellular trafficking, secretion and vesicular transport; (T) Defense mechanisms. COGs, Clusters of Orthologous Groups; WT, wild type; LP, Lactobacillus plantarum LP-Onlly; IL, interleukin; IL-10 ${ }^{-/-}$, IL-10 knock out.

During the metagenome analysis, clean reads were mapped to microbial reference genomes. The principal component analysis clearly differentiated the IL $10^{-/}+$LP group and WT + LP group; however, the samples of IL-10 ${ }^{-/-}$are scattered. 
This may be because inflammation of colitis influences the abundance and diversity of microbiota. The abundance of A. muciniphila and $P$. distasonis was significantly increased in IL-10 $1 /-$ LP group compared with the WT + LP group. The abundance of $B$. uniformis and $P$. distasonis was decreased in the IL- $10^{-/}$group. A previous study revealed that the increase of $A$. muciniphila was harmful to IBD and that $B$. uniformis has been identified as an essential member of gut microbiota (34). However, a previous study demonstrated that $P$. distasonis may be beneficial to IBD as it prevents intestinal inflammation in murine models (31). In addition, taxonomic analysis revealed that Firmicutes and Bacteroidetes are dominated in mice guts, which is consistent with previous studies. The Firmicutes/Bacteroidetes ratio increases in IL-10 ${ }^{-/-}$, and following LP administration this ratio decreased to a normal level when compared with the ratio of WT. However, this ratio increased markedly in the $\mathrm{WT}+\mathrm{LP}$ group. The results demonstrate that alterations to the microbiota composition may serve an important role in IBD, and the administration of LP may regulate the abundance and diversity of gut microbiota.

In the analysis of functional activities, the relative abundance of COGs was observed to change in IL-10/- mice. The function categories of COG, including 'Cell cycle control, cell division', 'Amino acid transport and metabolism', 'Carbohydrate transport and metabolism', 'Transcription' and 'Replication, recombination and repair' were significantly influenced. The 'Carbohydrate and nucleotide metabolism', 'Lipid and amino acid metabolism' and 'Amino acid transport and metabolism' were found to be associated with IBD in a recent study (5).

In conclusion, the present study further demonstrated the effectiveness of LP in the treatment of colitis. The current study provided an overview of gut microbiota components of colitis, and revealed the ability of LP to regulate the gut microbiota, which may be important in attenuating the inflammation of colitis. However, further investigations regarding LP and gut microbiota in colitis are required to reveal how metabolic changes to LP attenuates the colitis, and how the host immune system interacts with LP.

\section{Acknowledgements}

The present study was supported by National Natural Science Foundation of China (grant no. 81200264).

\section{References}

1. Guarner F and Malagelada JR: Gut flora in health and disease. Lancet 361: 512-519, 2003.

2. Ussar S, Griffin NW, Bezy O, Fujisaka S, Vienberg S, Softic S, Deng L, Bry L, Gordon JI and Kahn CR: Interactions between gut microbiota, host genetics and diet modulate the predisposition to obesity and metabolic syndrome. Cell Metab 22: 516-530, 2015.

3. Abraham $\mathrm{C}$ and Cho JH: Inflammatory bowel disease. $\mathrm{N}$ Engl $\mathrm{J}$ Med 361: 2066-2078, 2009.

4. Scaldaferri F, Gerardi V, Lopetuso LR, Del Zompo F, Mangiola F, Boškoski I, Bruno G, Petito V, Laterza L, Cammarota G, et al: Gut microbial flora, prebiotics, and probiotics in IBD: Their current usage and utility. Biomed Res Int 2013: 435268, 2013.

5. Hansen JJ: Immune responses to intestinal microbes in inflammatory bowel diseases. Curr Allergy Asthma Rep 15: 61, 2015.

6. Rup L: The human microbiome project. Indian J Microbiol 52 $315,2012$.
7. Gevers D, Knight R, Petrosino JF, Huang K, McGuire AL, Birren BW, Nelson KE, White O, Methé BA and Huttenhower C: The human microbiome project: A community resource for the healthy human microbiome. PLoS Biol 10: e1001377, 2012.

8. Ott SJ, Musfeldt M, Wenderoth DF, Hampe J, Brant O, Fölsch UR, Timmis KN and Schreiber S: Reduction in diversity of the colonic mucosa associated bacterial microflora in patients with active inflammatory bowel disease. Gut 53: 685-693, 2004

9. Morgan XC, Tickle TL, Sokol H, Gevers D, Devaney KL, Ward DV, Reyes JA, Shah SA, LeLeiko N, Snapper SB, et al: Dysfunction of the intestinal microbiome in inflammatory bowel disease and treatment. Genome Biol 13: R79, 2012.

10. Sokol H, Lay C, Seksik P and Tannock GW: Analysis of bacterial bowel communities of IBD patients: What has it revealed? Inflamm Bowel Dis 14: 858-867, 2008.

11. Swidsinski A, Ladhoff A, Pernthaler A, Swidsinski S, Loening-Baucke V, Ortner M, Weber J, Hoffmann U, Schreiber S, Dietel $\mathrm{M}$ and Lochs $\mathrm{H}$ : Mucosal flora in inflammatory bowel disease. Gastroenterology 122: 44-54, 2002.

12. Petschow B, Doré J, Hibberd P, Dinan T, Reid G, Blaser M, Cani PD, Degnan FH, Foster J, Gibson G, et al: Probiotics, prebiotics, and the host microbiome: The science of translation. Ann N Y Acad Sci 1306: 1-17, 2013

13. Rembacken BJ, Snelling AM, Hawkey PM, Chalmers DM and Axon AT: Non-pathogenic Escherichia coli versus mesalazine for the treatment of ulcerative colitis: A randomised trial. Lancet 354: 635-639, 1999.

14. Prantera $\mathrm{C}$ and Scribano ML: Probiotics and Crohn's disease. Dig Liver Dis 34 (Suppl 2): S66-S67, 2002.

15. Hedin C, Whelan K and Lindsay JO: Evidence for the use of probiotics and prebiotics in inflammatory bowel disease: A review of clinical trials. Proc Nutr Soc 66: 307-315, 2007.

16. Gibson GR: Dietary modulation of the human gut microflora using the prebiotics oligofructose and inulin. J Nutr 129 (7 Suppl): 1438S-1441S, 1999.

17. Sartor RB: Therapeutic manipulation of the enteric microflora in inflammatory bowel diseases: Antibiotics, probiotics, and prebiotics. Gastroenterology 126: 1620-1633, 2004.

18. Everard A, Lazarevic V, Gaïa N, Johansson M, Ståhlman M, Backhed F, Delzenne NM, Schrenzel J, François P and Cani PD: Microbiome of prebiotic-treated mice reveals novel targets involved in host response during obesity. ISME J 8: 2116-2130, 2014.

19. Xia Y, Chen HQ, Zhang M, Jiang YQ, Hang XM and Qin HL: Effect of Lactobacillus plantarum LP-Onlly on gut flora and colitis in interleukin-10 knockout mice. J Gastroenterol Hepatol 26: 405-411, 2011.

20. Human Microbiome Project Consortium: A framework for human microbiome research. Nature 486: 215-221, 2012.

21. Human Microbiome Project Consortium: Structure, function and diversity of the healthy human microbiome. Nature 486: 207-214, 2012.

22. Zerbino DR and Birney E: Velvet: Algorithms for de novo short read assembly using de Bruijn graphs. Genome Res 18: 821-829, 2008.

23. Zhu W, Lomsadze A and Borodovsky M: Ab initio gene identification in metagenomic sequences. Nucleic Acids Res 38: e132, 2010.

24. Huerta-Cepas J, Szklarczyk D, Forslund K, Cook H, Heller D, Walter MC, Rattei T, Mende DR, Sunagawa S, Kuhn M, et al: eggNOG 4.5: A hierarchical orthology framework with improved functional annotations for eukaryotic, prokaryotic and viral sequences. Nucleic Acids Res 44: D286-D293, 2016.

25. Edgar RC: MUSCLE: Multiple sequence alignment with high accuracy and high throughput. Nucleic Acids Res 32: 1792-1797, 2004.

26. Sinha S and Lynn AM: HMM-ModE: Implementation, benchmarking and validation with HMMER3. BMC Res Notes 7: 483, 2014.

27. Dray $\mathrm{S}$ and Dufour AB: The ade4 package: Implementing the duality diagram for ecologists. J Stat Softw 22: 20, 2007.

28. Wixon J and Kell D: The Kyoto encyclopedia of genes and genomes-KEGG. Yeast 17: 48-55, 2000.

29. Markowitz VM: Microbial genome data resources. Curr Opin Biotechnol 18: 267-272, 2007.

30. Ganesh BP, Klopfleisch R, Loh G and Blaut M: Commensal Akkermansia muciniphila exacerbates gut inflammation in Salmonella Typhimurium-infected gnotobiotic mice. PLoS One 8: e74963, 2013 
31. Kverka M, Zakostelska Z, Klimesova K, Sokol D, Hudcovic T, Hrncir T, Rossmann P, Mrazek J, Kopecny J, Verdu EF and Tlaskalova-Hogenova $\mathrm{H}$ : Oral administration of Parabacteroides distasonis antigens attenuates experimental murine colitis through modulation of immunity and microbiota composition. Clin Exp Immunol 163: 250-259, 2011.

32. Qin J, Li R, Raes J, Arumugam M, Burgdorf KS, Manichanh C, Nielsen T, Pons N, Levenez F, Yamada T, et al: A human gut microbial gene catalogue established by metagenomic sequencing. Nature 464: 59-65, 2010.
33. Fenner L, Roux V, Mallet MN and Raoult D: Bacteroides massiliensis sp. nov., isolated from blood culture of a newborn. Int J Syst Evol Microbiol 55: 1335-1337, 2005.

34. Png CW, Linden SK, Gilshenan KS, Zoetendal EG, McSweeney CS, Sly LI, McGuckin MA and Florin TH: Mucolytic bacteria with increased prevalence in IBD mucosa augment in vitro utilization of mucin by other bacteria. Am J Gastroenterol 105: 2420-2428, 2010. 